\title{
OPEN Relationship between continuity of care and clinical outcomes in patients with dyslipidemia in Korea: a real world claims database study
}

Juhee Lee ${ }^{1,7}$, Eunyoung Choi ${ }^{2,3,7}$, Eunjung Choo ${ }^{4}$, Siachalinga Linda ${ }^{2}$, Eun Jin Jang ${ }^{5 \bowtie} \&$ lyn-Hyang Lee ${ }^{2,6 \bowtie}$

Dyslipidemia is a risk factor for atherosclerotic cardiovascular disease and requires proactive management. This study aimed to investigate the association between care continuity and the outcomes of patients with dyslipidemia. We conducted a retrospective cohort study on patients with dyslipidemia by employing the Korea National Health Insurance claims database during the period 2007-2018. The Continuity of Care Index (COCI) was used to measure continuity of care. We considered incidence of atherosclerotic cardiovascular disease as a primary outcome. A Cox's proportional hazards regression model was used to quantify risks of primary outcome. There were 236,486 patients newly diagnosed with dyslipidemia in $\mathbf{2 0 0 8}$ who were categorized into the high and low COC groups depending on their $\mathrm{COCl}$. The adjusted hazard ratio for the primary outcome was 1.09 times higher (95\% confidence interval: 1.06-1.12) in the low COC group than in the high COC group. The study shows that improved continuity of care for newly-diagnosed dyslipidemic patients might reduce the risk of atherosclerotic cardiovascular disease.

Dyslipidemia refers to imbalance in the levels of one or more kinds of lipids such as total cholesterol, low-density lipoprotein cholesterol, triglycerides and high-density lipoprotein cholesterol in the blood ${ }^{1}$, and is a well-known risk factor for atherosclerotic cardiovascular disease (ASCVD) $)^{2,3}$. ASCVD is increasing worldwide and is among the leading causes of mortality and morbidity. Since dyslipidemia has been reported to be a leading cause of ASCVD, early management of dyslipidemia is a prerequisite to preventing ASCVD ${ }^{3-7}$. Several guidelines suggest that the management of lipid modification in the presence of dyslipidemia is important ${ }^{3,7,8}$. However, dyslipidemia seldom has distinct subjective symptoms nor induce discomfort that patients may be aware of ${ }^{9}$, thus, the awareness of the disease is low ${ }^{10}$, resulting into rates of treatment initiation and adherence to be low. Although health behavior intervention is highly recommended ${ }^{7}$, self-management is likely to be ineffective due to lack of patient awareness or commitment ${ }^{11}$. Accordingly, to reduce obstacles to treatment for silent chronic diseases like dyslipidemia, the role of medical staff can be significant ${ }^{12}$. In this regard, the existing literature suggests that continuity of care (COC) between health providers and patients in chronic conditions might improve clinical outcomes $^{13-16}$. COC is defined as 'the degree to which a series of discrete healthcare events is experienced as coherent, and connected and consistent with the patient's medical needs and personal context ${ }^{17}$. The literature indicates that improved continuity of care might lower mortality ${ }^{15}$, hospitalization rates ${ }^{16}$, emergency department $\operatorname{costs}^{13}$, and medical expenses ${ }^{14}$ in different kinds of care settings or diseases.

Some studies have shown that high COC could reduce hospitalization rates, emergency room visits, and medical costs in hypertensive ${ }^{18}$ or heart failure patients ${ }^{19}$. However, little is known about the impact of COC in patients with dyslipidemia although the burden of dyslipidemia is growing. Among South Koreans older than

\footnotetext{
${ }^{1}$ Department of Statistics, Kyungpook National University, Daegu 41566, Republic of Korea. ${ }^{2}$ College of Pharmacy, Yeungnam University, Gyeongsan 38541, Republic of Korea. ${ }^{3}$ Department of Pharmacy, Ulsan University Hospital, Ulsan 44033, Republic of Korea. ${ }^{4}$ College of Pharmacy, Ajou University, Suwon 16499, Republic of Korea. ${ }^{5}$ Department of Information Statistics, Andong National University, Andong 36729, Republic of Korea. ${ }^{6}$ Department of Health Sciences, University of York, York YO10 5DD, UK. ${ }^{7}$ These authors contributed equally: Juhee Lee and Eunyoung Choi. ${ }^{\circledR}$ email: ejjang@anu.ac.kr; leeiynhyang@ynu.ac.kr
} 


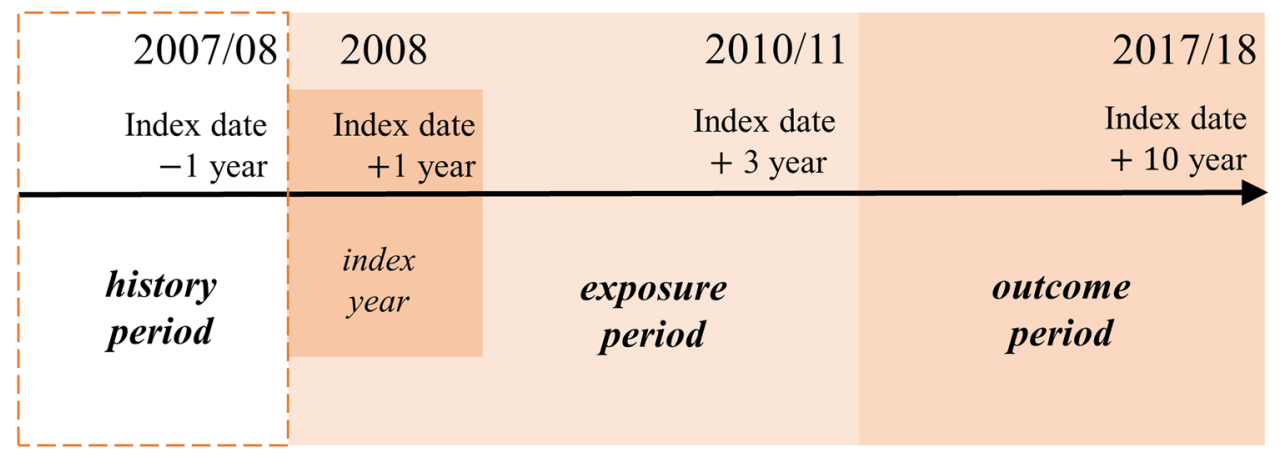

Figure 1. The schematic diagram of the time frame for study.

thirty years, 4 out of 10 were diagnosed with dyslipidemia during the $2010 \mathrm{~s}^{20,21}$. The prevalence of hyperlipidemia, a subtype of dyslipidemia, doubled from $13.4 \%$ in 2010 to $21.4 \%$ in $2019^{22}$. Between 2010 and 2019, deaths from hyper-LDL-cholesterolemia increased from 12,000 to 15,000 in South Korea and from 3.75 million to 4.40 million worldwide among those older than thirty ${ }^{23}$. Thus in this study, we investigated the association between COC and the outcomes of patients with dyslipidemia. It was hypothesized that a high COC would be related with positive clinical outcomes in dyslipidemic patients.

\section{Methods}

Data sources. Claims data for analysis, which included all patients $(n=1,590,501)$ newly diagnosed with dyslipidemia in 2008, were provided by the Korean National Health Insurance Service (NHIS). The Korea National Health Insurance database contains all claims data for the Korean residents covered by National Health Insurance (NHI) and Medical Aid (MAid) ${ }^{24}$. The NHIS claims data are provided to academic researchers after de-identification of personal information. The data for this study contained de-identified patient demographic information, diagnoses, diagnostic tests, utilization of medical facilities and prescriptions of patient, death records and level of NHI contributions between 2006 and $2018^{25}$.

Study design and timing. This is a retrospective cohort study. This study was conducted in accordance with the STROBE guideline ${ }^{26}$. Figure 1 displays the study period between 2007 and 2018 and explains each period. We set 2008 as the index year for defining the patients with dyslipidemia. The index date was set as the earliest date in 2008 of dyslipidemia diagnosis for a patient identified for this study. The history period was defined as 1 year before the index date. To avoid time-dependent bias, we independently set the exposure and outcome period in the primary analysis. The exposure period was defined as the first 3 years after the index date and the outcome period was defined as the following 7 years. We restructured available data on an annual basis from the index date. As a result, the same duration was applied to all study subjects, although the actual observation period varied. For example, in the case of a patient identified on 1 January 2008, the observation period was from 1 January 2008 to 31 December 2017, whereas for a patient identified on 1 May 2008 the observation period continued until 30 April 2018. Each patient was followed from the end of the exposure period until death, an event, or end of data availability, whichever occurred first.

Study population. We identified patients newly diagnosed with dyslipidemia, which was coded as E78.0 E78.9 according to the International Classification of Diseases, 10th version (ICD-10) ${ }^{27}$, that made at least two ambulatory visits during the one year after the index date, and at least four visits during the first three years (the exposure period) $)^{16,28,29}$. Those diagnosed with hypertensive diseases (coded I10 I13, I15), ischemic heart disease (I20 I25), cerebrovascular diseases and related syndromes (I60 I69, G45 G46), diabetes mellitus with circulatory complications (E10.5, E11.5, E12.5, E13.5, E14.5) and cancer (C00 C97) from January 1, 2006 to the index date were excluded because such diseases could either be related with the study outcome or affect healthcare use behavior. Those with missing personal information (e.g., age or sex) were 1,103 (0.44\%) and excluded from the data. Those diagnosed with myocardial infarction (I21.0-4, I21.9, I22.0-1, I22.8-9), stable or unstable angina (I20), ischemic stroke (I63.0-6, I63.8-9), and transient ischemic attack (G45.0-3, G45.8-9), or died during the exposure period were also excluded. The selection process of study population is shown in Fig. 2.

Measures. Continuity of care. To measure COC as an exposure variable, we used the Bice-Boxerman Continuity of Care Index $(\mathrm{COCI})^{28}$, which is recommended for use in the context of South Korea, where patients may potentially contact many different healthcare providers. The equation used to calculate COCI is as follows:

$$
\mathrm{COCI}=\frac{\sum_{j=1}^{M} n_{j}^{2}-N}{N(N-1)},
$$

where $N$ is the total number of ambulatory care visits, $n_{j}$ is the number of visits to doctor $j$, and $M$ is the total number of doctors that a patient has met. The COC index ranges from 0 to 1 . If all of the visits made by a patient 


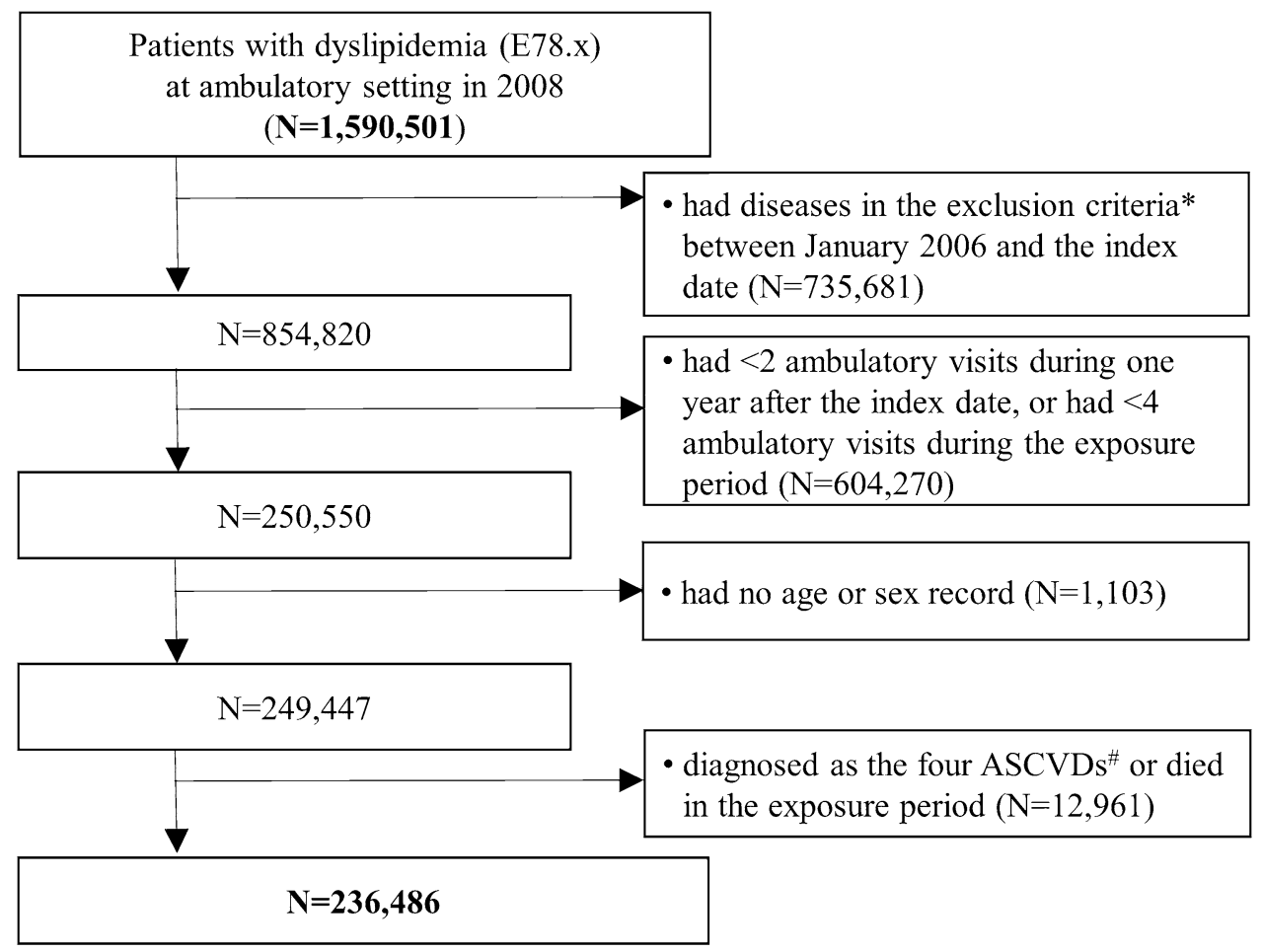

Figure 2. Selection of study population. ${ }^{*}$ Diseases in the exclusion criteria included hypertensive diseases (I10 I13, I15), ischemic heart diseases (I20 I25), cerebrovascular diseases and related syndromes (I60 I69, G45 G46), diabetes mellitus with circulatory complications (E10.5, E11.5, E12.5, E13.5, E14.5), and cancer (C00 C97). "The four ASCVDs included myocardial infarction (I21.0-4, I21.9, I22.0-1, I22.8-9), stable or unstable angina (I20), ischemic stroke (I63.0-6, I63.8-9), and transient ischemic attack (G45.0-3, G45.8-9).

are to the same doctor, the index equals 1 . If each visit involves a different doctor, the index equals 0 . Thus, a higher COCI indicates better continuity of care. We regarded a visit to the same doctor's office as a visit to the same doctor in a primary care setting and a visit to the same medical department in the same hospital as a visit to the same doctor in a secondary care setting.

Continuity cohort. As far as we are aware, there is no generally used cut-off value for high and low COCI. Thus, we set the cut-off at 0.8 and defined a COCI of $\geq 0.8$ as high and a COCI of $<0.8$ as low based on the first 3 -year COCI distribution of our study subjects from an interim analysis. In the interim analysis, patients with a COCI of $\geq 0.8$ accounted for $53.8 \%$ of all study subjects (Fig. 3 ). The COCI would be 0.8 , if a patient made ten doctor visits that included nine visits to the same doctor.

Outcomes. We defined a primary outcome as the diagnosis of one of the four diseases, namely, myocardial infarction, stable or unstable angina, ischemic stroke, and transient ischemic attack during the outcome period. These ASCVDs were operationally defined as; (i) myocardial infarction, diagnosed I21.0-4, I21.9, I22.0-1, I22.8-9 and treated using Percutaneous Coronary Intervention (PCI), Coronary Artery Bypass Surgery (CABG), or fibrinolytics, (ii) stable or unstable angina, diagnosed I20 and treated using PCI, CABG, or fibrinolytics, (iii) ischemic stroke, diagnosed I63.0-6, I63.8-9 by brain imaging (CT or MRI), and (iv) transient ischemic attack, diagnosed G45.0-3, G45.8-9 by brain imaging (CT or MRI).

Secondary outcomes were defined as health services utilization and related medical costs. Health services utilization included the number of patients with one of the four ASCVD diseases, related hospitalization and their average length of hospitalization, and number of patients visiting the emergency departments (EDs) due to one of the four ASCVD diseases and their average frequency. Related medical costs included expenses incurred by dyslipidemia and the four ASCVDs and were classified as public and out-of-pocket expenditures.

Covariates. Covariates included individual characteristics such as sex, age, insurance contributions, payer, and urbanization level of residence at the index date. Insurance contributions were classified into three categories (high, moderate, and low). In Korea, there are two types of payers: NHI and MAid and about $97 \%$ of the population is covered by NHI and $3 \%$ by MAid $^{30}$. The urbanization level was classified into three categories (large urban, small urban, and rural). In addition, Elixhauser comorbidity indices (ECIs) were computed as a proxy of patient health status ${ }^{31}$ based on diagnoses from outpatient and inpatient records during the history period. In addition, we also considered whether patients had a diagnosis of diabetes or were prescribed antihyperlipidemic 


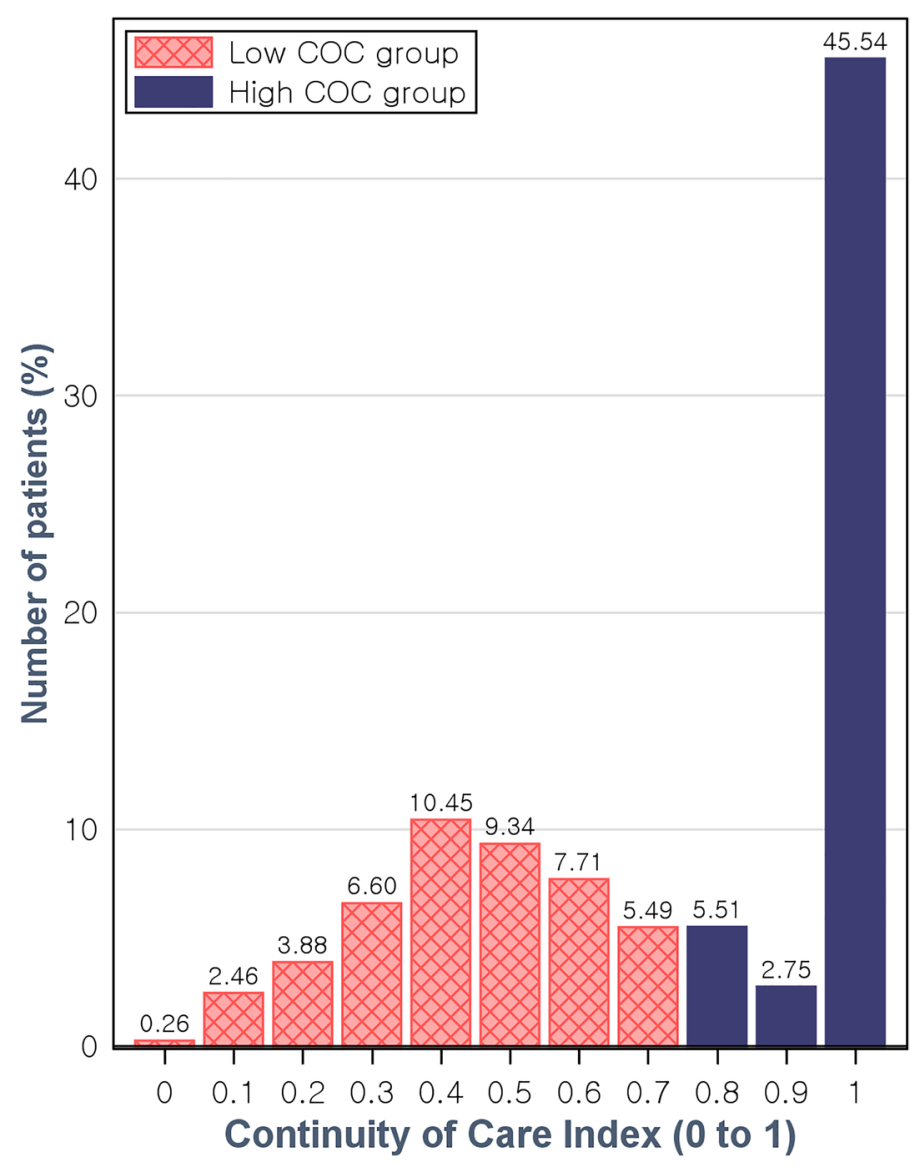

Figure 3. Continuity of Care Index distribution during the first 3 years. COC continuity of care.

agent. For reference purposes, we also collected baseline data about health services utilization and costs regardless of the disease diagnosis.

Statistical analysis. Patient characteristics, health services utilization at baseline, COCI, and study outcomes are summarized as means and standard deviations (SDs) or medians and interquartile ranges (IQRs) for continuous variables and as frequencies and percentages for categorical variables. To compare patient characteristics by level of continuity of care, we performed the Kruskal-Wallis test for continuous variables because the assumption of normality was not satisfied. The Chi-squared test was performed to analyze categorical variables.

The association between $\mathrm{COC}$ and health outcome was investigated using the Cox proportional hazard regression model. The proportional assumption of the Cox proportional hazards model was checked by examining the cumulative Martingale residuals plots and the Kolmogorov-type supremum test. A Kaplan-Meier survival plot of the four ASCVDs was constructed and the p-value from the log-rank test was presented. Unadjusted hazard ratios (HRs) and adjusted HRs (adjusted for age, sex, insurance contribution group, living area, Elixhauser comorbidity index, and antihyperlipidemic agent use) were presented with $95 \%$ confidence intervals (CIs). Individuals whose information about insurance contribution and living area were missing were not excluded, and missing value was considered as an independent category in the statistical regression model. For sensitivity analysis, COCI was considered as a time-dependent exposure in the additional model, as the initial COC is likely to change over time. The subgroup analyses for the association between COC and health outcome were performed according to patient characteristics and the number of visits during the first 3 years.

The statistical analysis was performed using the SAS statistical software package (version 9.4, SAS Institute, Cary, NC, USA) and a p-value $<0.05$ was considered statistically significant.

Ethics declarations. This study was performed in accordance with the Declaration of Helsinki and was approved by the Institutional Review Board of Yeungnam University (YU201804004002). Written informed consent was waived because this study analyzed anonymous claims data provided for research purposes by the Korean National Health Insurance Service. 
General characteristics

\begin{tabular}{|c|c|c|c|c|}
\hline \multirow{2}{*}{ Sex } & Women & $65,778(51.70)$ & $63,502(58.13)$ & \multirow{2}{*}{$<0.001$} \\
\hline & Men & $61,460(48.30)$ & $45,746(41.87)$ & \\
\hline \multirow{3}{*}{ Age } & Median (IQR) & $50(42-58)$ & $51(44-59)$ & $<0.001$ \\
\hline & Mean \pm SD & $49.87 \pm 12.41$ & $51.27 \pm 11.65$ & \\
\hline & $\geq 65$ years & $15,500(12.18)$ & $14,508(13.28)$ & $<0.001$ \\
\hline \multirow{3}{*}{ Insurance contributions $\mathrm{s}^{\mathrm{a}}$} & High & $58,746(46.17)$ & $49,443(45.26)$ & \multirow{3}{*}{$<0.001$} \\
\hline & Moderate & $36,809(28.93)$ & $30,921(28.30)$ & \\
\hline & Low & $24,660(19.38)$ & $22,084(20.21)$ & \\
\hline \multirow{2}{*}{ Payer } & NHI & $121,803(95.73)$ & $104,059(95.25)$ & \multirow{2}{*}{$<0.001$} \\
\hline & MAid & $5,435(4.27)$ & $5,189(4.75)$ & \\
\hline \multirow{3}{*}{ Urbanization level of residence ${ }^{b}$} & Large urban & $63,655(50.03)$ & $54,045(49.47)$ & \multirow{3}{*}{$<0.001$} \\
\hline & Small urban & $53,151(41.77)$ & $44,820(41.03)$ & \\
\hline & Rural & $10,310(8.10)$ & $10,262(9.39)$ & \\
\hline \multirow{5}{*}{ Elixhauser comorbidity index } & Median (IQR) & $1(0-2)$ & $1(0-2)$ & 0.110 \\
\hline & 0 & $56,976(44.78)$ & $48,755(44.63)$ & \multirow{4}{*}{$<0.001$} \\
\hline & 1 & $38,029(29.89)$ & $32,617(29.86)$ & \\
\hline & 2 & $20,122(15.81)$ & $16,857(15.43)$ & \\
\hline & $3+$ & $12,111(9.52)$ & $11,019(10.09)$ & \\
\hline \multirow{2}{*}{ Comorbidity } & Diabetes mellitus & $19,350(15.21)$ & $12,875(11.79)$ & \multirow{2}{*}{$<0.001$} \\
\hline & None & $107,888(84.79)$ & $96,373(88.21)$ & \\
\hline \multirow{2}{*}{ Antihyperlipidemic agent use } & Yes & $38,484(30.25)$ & $34,962(32.00)$ & \multirow{2}{*}{$<0.001$} \\
\hline & No & $88,754(69.75)$ & $74,286(68.00)$ & \\
\hline
\end{tabular}

Health services utilization for all diseases (annual average during the exposure period)

\begin{tabular}{|c|c|c|c|c|}
\hline \multirow{6}{*}{ Ambulatory visits } & Median (IQR) & $16.33(10.33-25.67)$ & $19(11.67-30.00)$ & $<0.001$ \\
\hline & Mean \pm SD & $20.91 \pm 18.19$ & $24.36 \pm 20.93$ & \\
\hline & $4-8$ & $51,954(40.83)$ & $50,175(45.93)$ & \multirow{4}{*}{$<0.001$} \\
\hline & $9-14$ & $27,105(21.30)$ & $24,418(22.35)$ & \\
\hline & $15-23$ & $21,648(17.01)$ & $19,137(17.52)$ & \\
\hline & $24+$ & $26,531(20.85)$ & $15,518(14.20)$ & \\
\hline \multirow{2}{*}{ Pharmacy visits } & Median (IQR) & $13.33(8.33-20.33)$ & $14.67(9.33-22.67)$ & $<0.001$ \\
\hline & Mean \pm SD & $15.83 \pm 11.55$ & $17.77 \pm 12.95$ & \\
\hline \multirow{6}{*}{$\begin{array}{l}\text { Annual average all medical costs } \\
(1000 \mathrm{KRW})^{\mathrm{c}}\end{array}$} & Overall, median (IQR) & $1119(654-1810)$ & $1200(714-2002)$ & $<0.001$ \\
\hline & Public expenditure & $762(440-1263)$ & $820(481-1411)$ & $<0.001$ \\
\hline & $\begin{array}{l}\text { Out-of-pocket pay- } \\
\text { ment }\end{array}$ & $327(181-533)$ & $349(200-574)$ & $<0.001$ \\
\hline & Overall, mean \pm SD & $1566 \pm 2182$ & $1732 \pm 2229$ & \\
\hline & Public expenditure & $1147 \pm 1917$ & $1275 \pm 1915$ & \\
\hline & $\begin{array}{l}\text { Out-of-pocket pay- } \\
\text { ment }\end{array}$ & $417 \pm 388$ & $454 \pm 427$ & \\
\hline
\end{tabular}

Table 1. Patient characteristics by level of continuity of care. COC continuity of care, IQR interquartile

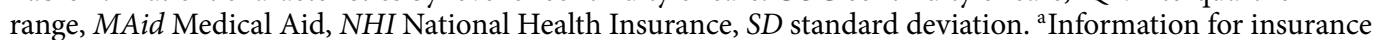
contribution was missing for 7023 (5.5\%) patients in the high COC group and $6800(6.2 \%)$ patients in the low COC group. ${ }^{b}$ Information for urbanization level of residence was missing for $122(0.1 \%)$ patients in the high COC group and $121(0.1 \%)$ patients in the low COC group. ${ }^{c} 1$ US dollar $=1200 \mathrm{KRW}$ in Aug 2021. $P$-values were calculated by Kruskal-Wallis tests for continuous variables and by Chi-squared tests for categorical variables.

\section{Results}

Study population. Table 1 shows the baseline characteristics of patients by continuity group. The study population included 236,486 patients, of which $53.8 \%$ were in the high COC group and $46.2 \%$ were in the low COC group. Generally, although there were more women in both groups, women were more prevalent in the low COC group (58.13\%). There was relatively a small difference in the proportion of men and women in the high COC group (48.30\% vs. 51.70\%). Those in the low COC group were slightly older by about 1.5 years on average $(\mathrm{p}<0.001)$ and more likely to reside in rural areas $(9.39 \%$ vs. $8.10 \%)$. While insurance contributions and payer were significantly different $(\mathrm{p}<0.001)$, the absolute differences between the groups were $<1 \%$.

Average ECIs were similar in the two study groups, however, patients with 3 or more comorbidities were more likely to be in the low COC group and those with diabetes were more likely to be in the high COC group. 


\begin{tabular}{|c|c|c|c|c|}
\hline \multicolumn{2}{|l|}{ Variable } & $\begin{array}{l}\text { High COC group } \\
\mathbf{n}=127,238\end{array}$ & $\begin{array}{l}\text { Low COC group } \\
n=109,248\end{array}$ & $p$-value \\
\hline \multicolumn{5}{|c|}{ Exposure period ( 3 years) } \\
\hline \multirow{2}{*}{ COCI in all population } & Median (IQR) & \multicolumn{2}{|l|}{$0.87(0.50-1.00)$} & \\
\hline & Mean \pm SD & \multicolumn{2}{|l|}{$0.75 \pm 0.28$} & \\
\hline \multirow{2}{*}{ COCI by group } & Median (IQR) & $1.00(1.00-1.00)$ & $0.49(0.36-0.60)$ & $<0.001$ \\
\hline & Mean \pm SD & $0.98 \pm 0.05$ & $0.48 \pm 0.17$ & \\
\hline \multirow{4}{*}{ No. of visits } & Median (IQR) & $11(6-21)$ & $9(6-17)$ & $<0.001$ \\
\hline & Mean \pm SD & $15.40 \pm 14.50$ & $13.31 \pm 12.57$ & \\
\hline & Primary care & $11.97 \pm 15.65$ & $10.14 \pm 12.81$ & \\
\hline & $\begin{array}{l}\text { Secondary } \\
\text { care }\end{array}$ & $3.43 \pm 6.60$ & $3.18 \pm 5.23$ & \\
\hline \multicolumn{5}{|c|}{ Exposure plus outcome period (10 years) } \\
\hline \multirow{2}{*}{ COCI in all population } & Median (IQR) & \multicolumn{3}{|l|}{$0.58(0.39-0.90)$} \\
\hline & Mean \pm SD & \multicolumn{3}{|l|}{$0.62 \pm 0.27$} \\
\hline \multirow{2}{*}{ COCI by group } & Median (IQR) & $0.83(0.51-1.00)$ & $0.43(0.30-0.61)$ & $<0.001$ \\
\hline & Mean \pm SD & $0.75 \pm 0.25$ & $0.47 \pm 0.21$ & \\
\hline \multirow{4}{*}{ No. of visits } & Median (IQR) & $33(12-64)$ & $34(14-61)$ & 0.002 \\
\hline & Mean \pm SD & $44.62 \pm 44.34$ & $43.12 \pm 41.28$ & \\
\hline & Primary care & $33.94 \pm 45.07$ & $32.49 \pm 40.65$ & \\
\hline & $\begin{array}{l}\text { Secondary } \\
\text { care }\end{array}$ & $10.68 \pm 20.02$ & $10.63 \pm 18.08$ & \\
\hline
\end{tabular}

Table 2. COCI and number of visits to medical facilities by period. COC continuity of care, COCI continuity of care index, IQR interquartile range, $S D$ standard deviation. $P$-values were calculated by Kruskal-Wallis tests for continuous variables.

\begin{tabular}{|c|c|c|c|}
\hline Outcomes & High COC group $(\mathrm{n}=127,238)$ & Low COC group $(n=109,248)$ & $p$-value \\
\hline \multicolumn{4}{|l|}{ Risk for the four ASCVDs } \\
\hline No. of patients having the four ASCVDs (\%) & $6971(5.48)$ & $7279(6.66)$ & $<0.001$ \\
\hline \multicolumn{4}{|c|}{ Hazard ratio by standard Cox's hazards regression model } \\
\hline Unadjusted HR (95\% CI) & 1.0 (reference) & $1.23(1.10-1.16)$ & $<0.001$ \\
\hline Adjusted HR $(95 \% \mathrm{CI})^{\mathrm{a}}$ & 1.0 (reference) & $1.09(1.06-1.12)$ & $<0.001$ \\
\hline \multicolumn{4}{|c|}{ Hazard ratio by time-dependent model (sensitivity analysis) } \\
\hline Unadjusted HR (95\% CI) & 1.0 (reference) & $1.30(1.26-1.35)$ & $<0.001$ \\
\hline Adjusted HR $(95 \% \mathrm{CI})^{\mathrm{a}}$ & 1.0 (reference) & $1.27(1.23-1.31)$ & $<0.001$ \\
\hline
\end{tabular}

Table 3. Risks for the four ASCVDs from Cox's hazard regression models in Korean dyslipidemia patients. $A S C V D$ atherosclerotic cardiovascular disease, CI confidential interval, COC continuity of care, $H R$ hazard

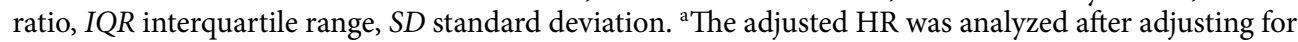
covariates including sex, age, insurance contribution, payer, urbanization level of residence, comorbidity as Elixhauser score and antihyperlipidemic agent use.

Patients on antihyperlipidemic agents in the index year were more likely to be in the low COC group. During the exposure period, patients in the low COC group used healthcare services more frequently than those in the high COC group.

Changes in continuity of care index. COCI decreased with time in both groups (Table 2). As the number of years increased from 3 to 10 years, the change in COC value was greater in the high COC group ( 1 to 0.83$)$ compared to the low COC group ( 0.49 to 0.43 ). The numbers of visits made in 3 and 10 years were greater in the high COC group and about three quarters of these visits were to primary care.

Risk for atherosclerotic cardiovascular diseases. Table 3 shows the risks for the four ASCVDs in the two study groups. After adjusting for covariates, the adjusted hazard ratio for the risk of the four ASCVDs was 1.09 (95\% CI 1.06-1.12) in the low COC group compared to the high COC group. In the sensitivity analysis using $\mathrm{COC}$ as a time-dependent exposure, the adjusted hazard ratio for the risk of the four ASCVDs was 1.27 (95\% CI 1.23-1.31) in the low COC group compared to the high COC group.

In Fig. 4, during the 7-year follow-up period, the probability of being event-free of the four ASCVDs were significantly lower in the low COC group $(\mathrm{p}<0.001)$. 


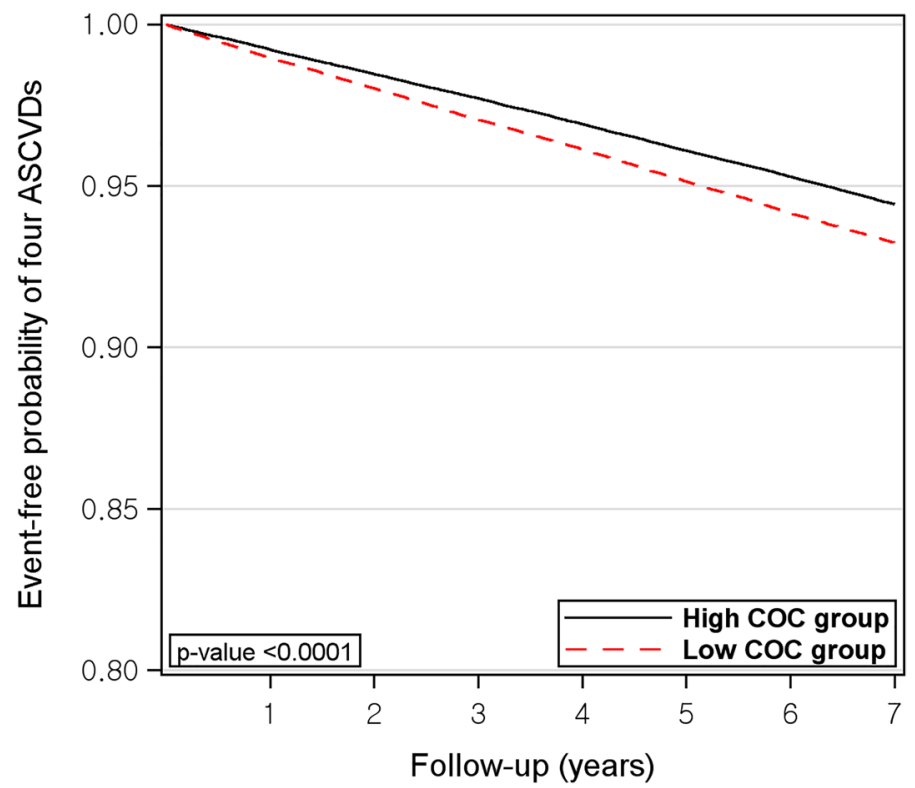

Figure 4. Event-free probability of four ASCVDs. ASCVD atherosclerotic cardiovascular disease, COC continuity of care.

\begin{tabular}{|c|c|c|c|}
\hline Outcomes & High COC group $(n=127,238)$ & Low COC group $(n=109,248)$ & $p$-value \\
\hline \multicolumn{4}{|c|}{ Health services utilization due to the four ASCVDs } \\
\hline No. of patients hospitalized (\%) & $5237(4.12)$ & $5375(4.92)$ & $<0.001$ \\
\hline $\begin{array}{l}\text { Average length of hospitalization } \\
\text { days per patient per year, median (IQR) }\end{array}$ & $10(4-24)$ & $9(3-23)$ & 0.001 \\
\hline No. of patients visiting ED (\%) & $2893(2.27)$ & $2853(2.61)$ & $<0.001$ \\
\hline $\begin{array}{l}\text { Frequency of visiting ED } \\
\text { per patient per year, median (IQR) }\end{array}$ & $1(1-1)$ & $1(1-1)$ & 0.558 \\
\hline \multicolumn{4}{|l|}{ Related medical costs } \\
\hline $\begin{array}{l}\text { Disease related medical costs }{ }^{\#} \\
1000 \text { KRW per patient per year, median (IQR) }\end{array}$ & $89(20-208)$ & $100(31-229)$ & $<0.001$ \\
\hline Public expenditure & $58(13-141)$ & $66(20-160)$ & $<0.001$ \\
\hline Out-of-pocket payment & $24(5-62)$ & $27(7-66)$ & $<0.001$ \\
\hline $\begin{array}{l}\text { Disease related medical costs }{ }^{\#} \\
1000 \text { KRW per patient per year, mean } \pm \text { SD }\end{array}$ & $315 \pm 1460$ & $347 \pm 1439$ & \\
\hline Public expenditure & $249 \pm 1250$ & $275 \pm 1236$ & \\
\hline Out-of-pocket payment & $65 \pm 249$ & $71 \pm 247$ & \\
\hline
\end{tabular}

Table 4. Health services utilization and related medical costs by level of continuity of care. ASCVD atherosclerotic cardiovascular disease, $C I$ confidential interval, $C O C$ continuity of care, ED Emergency department, $H R$ hazard ratio, $I Q R$ interquartile range, $S D$ standard deviation. ${ }^{a}$ Diseases related medical costs included costs for treating dyslipidemia and dyslipidemia related four ASCVDs; 1 US dollar $=1200 \mathrm{KRW}$ in Aug 2021. $P$-values were calculated by Kruskal-Wallis tests for continuous variables, and Chi-squared tests for categorical variables.

Related health services utilization and costs. Patients in the low COC group were more likely to be hospitalized or visit an ED due to one of the four ASCVDs and to have greater medical costs than those in the high COC group (Table 4). The frequency of ED visits per patient among those who had events was not significantly different between groups.

Subgroup analysis. The subgroup analysis demonstrated the same trend as the primary findings in regard to the risk for the four ASCVDs in most attributes (Fig. 5). However, the risk differences between the two groups were not statistically significant seemingly due to the small number of samples in the following groups; elderly, patients in MAid, those in rural area, those with ECI greater than 3, those who took antilipidemic agents, and those who made 15-23 doctor visits during the first 3 years. 
Subgroup

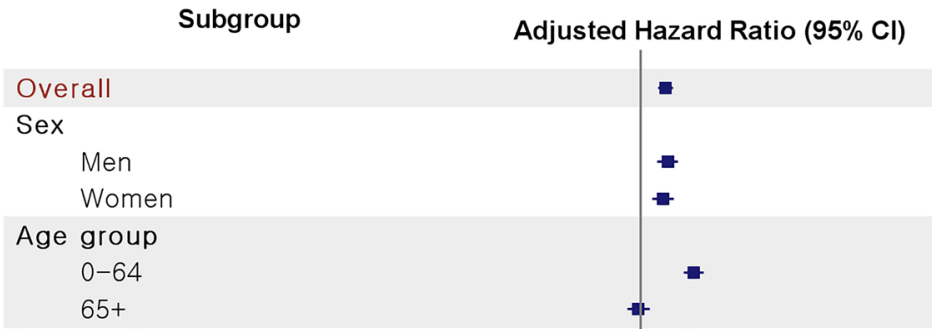

Payer

$\mathrm{NHI}$

MAid

Insurance contribution

High

Moderate

Low

Urbanization level of residence

Large urban

Small urban

Rural

Elixhauser cormorbidity index

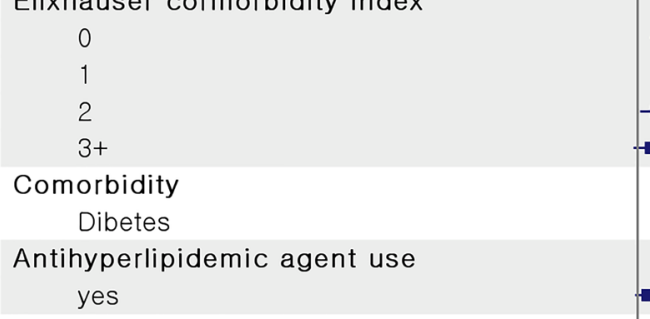

No. of visits in the first 3 years

4-8

$9-14$

$15-23$

$24+$
No. of patients with ASCVD

Low COC (\%) High COC (\%)

patients

$1.10(1.07-1.13)$

$7,279(6.7)$

$6.971(5.5)$

236,486

$1.11(1.07-1.15)$

$1.09(1.05-1.13)$

$3,394(7.4)$

$3,719(6.1)$

107,206

$3,885(6.1)$

$3,252(4.9)$

129,280

$1.21(1.17-1.25)$

$0.99(0.95-1.04)$

$5,243(5.5)$

$4,994(4.5)$

206,478

$2,036(14.0)$

$1,977(12.8)$

30,008

$1.11(1.08-1.14)$

$6.747(6.5)$

$6,509(5.3)$

225,862

$1.00(0.91-1.09)$

$532(10.3)$

$462(8.5)$

10,624

$1.09(1.05-1.14)$

$1.12(1.07-1.18)$

$3,113(6.3)$

$3,146(5.4)$

108,189

2,040 (6.6)

$1,891(5.1)$

$1,389(5.6)$

67,730

$1.12(1.06-1.19)$

$1,483(6.7)$

$3,146(4.9)$

46,744

\section{$1.10(1.06-1.14)$}

$3,226(6.0)$

$3,007(5.7)$

117,700

$1.11(1.06-1.15)$

$1.06(0.99-1.15)$

$3,062(6.8)$

$814(7.9)$

97,971

985 (9.6)

20,572

\section{$1.10(1.05-1.15)$}

$2,808(5.8)$

$2,793(4.9)$

$2,203(6.8)$

$2,023(5.3)$

105,731

$1.14(1.08-1.19)$

$1,261(7.5)$

$1,231(6.1)$

70,646

$1.08(1.01-1.15)$

$1,007(9.1)$

$924(7.6)$

36,979

$1.05(0.98-1.13)$

$1.13(1.06-1.20)$

$1,156(9.0)$

$1,440(7.4)$

32,225

$1.04(0.99-1.09)$

$2,365(6.8)$

$2,386(6.2)$

73,446

$1.15(1.10-1.20)$

$3,040(6.1)$

$2,322(4.5)$

102,129

$1.06(1.00-1.12)$

$1,656(6.8)$

$1,558(5.7)$

51,523

$1.01(0.95-1.08)$

$1,320(6.9)$

$1,321(6.1)$

40,785

$1.13(1.06-1.20)$

$1,263(8.1)$

$1,770(6.7)$

42,049

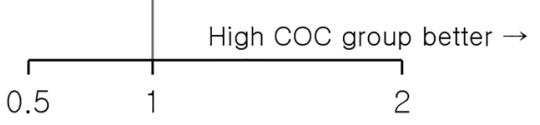

Figure 5. Subgroup analysis for the association between continuity of care and the risk for the four ASCVDs. $A S C V D$ atherosclerotic cardiovascular disease, CI confidence interval, COC continuity of care, MAid Medical Aid, NHI National Health Insurance.

\section{Discussion}

The results of this study show that a low COC level in newly diagnosed dyslipidemic patients is associated with increased risk of myocardial infarction, stable or unstable angina, ischemic stroke, or transient ischemic attack. From the perspective of a medical staff, patients in the high COC group may be interacted with continuously, and thus, staff are more likely to provide good quality of care (e.g., patient-tailored care) ${ }^{32}$. From the patient's point of view, they are more likely to be satisfied with medical staff, which might improve management and inter-personal trust ${ }^{33}$, and probably improve clinical results.

The study also demonstrates that a high COC level might have positive impact on health resources utilization and medical costs. However, it may be difficult to merely ascribe medical costs savings to the impact of high COC since patients with low COC already had higher medical costs in the first three years. Future studies are needed to determine the actual magnitude of cost savings. In previous studies, women, the elderly ${ }^{34-36}$ and patients further away from medical institutions ${ }^{35}$ tended to change doctors more frequently, resulting in low COC levels. The same phenomena were observed in the present study, that is, the low COC group contained higher proportions of women, elderly, and patients living in rural areas. A low socioeconomic status may increase the possibility of negative outcomes by lowering the chances of receiving healthcare services ${ }^{37}$, though this was not obvious with patients in the low COC group of the present analysis.

In the present study, the mean COCI among study subjects during the first 3 year period was 0.75 and decreased to 0.62 in the 10 year period. While no previous study has explored COC in patients with dyslipidemia, the distributions of COCI observed in two studies on patients with cardiovascular diseases compare well with our findings. Hong et al. found the mean COCI of 0.74 in Korean patients with hypertension in a 3-year period ${ }^{18}$. Another study by Vogt and colleagues, investigated COC in German patients with heart failure, reported that the mean COCI was 0.77 in a 2 year period ${ }^{19}$. There were some differences in studies for other diseases. The mean 
COCI in a 2 year span was reported to be 0.83 in Korean type 2 diabetes mellitus patients ${ }^{36}$, and 0.65 in Taiwanese COPD patients ${ }^{38}$. These demonstrated that the distribution of COCI is dependent on disease, measurement period, and country even for chronic diseases.

To the best of our knowledge, this study is the first to evaluate the effects of COC on patients with dyslipidemia using long-term real world data on the Korean population. In this study, exposure and outcome period were independently set in the primary analysis to avoid time-dependent bias ${ }^{39}$, and the sensitivity of the hazard ratios were further tested by using COCI as a time-dependent covariate. ASCVD is a chronic disease and takes a relatively long time to progress from dyslipidemia. In this regard, the present study was sufficiently long to explore the relationship between COC and clinical outcomes as it utilized 10 years of claims data.

However, the use of secondary data for analysis inherently introduces limitations. First, though sophisticated statistical methods were used to control for potential confounders, they may have failed to exclude the effects of some meaningful factors affecting patients' relationships with professionals such as occupation, education, or health behaviors, which are seldom included in claims data. Second, the claims data did not contain any information about individual doctors. To compensate for this, we considered visits to the same medical department in the same hospital in a secondary care setting as visits to the same provider, because most patients see the same doctor when they visit the same medical department in the same institution. Third, this study employed an empirical method to classify patients into the two continuity groups. There are no previous study suggesting a standard or optimal procedure for grouping patients based on COCI, rather median values ${ }^{36,40}$, tertiles ${ }^{41,42}$, and other metrics ${ }^{43,44}$ have been used. This may mean that the distributions and cut-offs of COC are research population dependent. Furthermore, the Korean healthcare system provides universal coverage, and patients have the right to choose a healthcare provider with few legal restrictions, which limits the external validity of this study.

The study findings suggest that policies improving care continuity between doctors and patients with dyslipidemia results in positive clinical outcomes. Future policies need to identify factors that hinder lasting quality relationships and make efforts to address them. Also, efforts should be made to ensure that doctors and patients are aware of the good impact of continuity of care. In this regard, qualitative study investigating the perceptions of professionals or patients on continuity of care will be helpful to disclose their awareness and views of it.

\section{Conclusion}

Improved continuity of care might decrease the risks of myocardial infarction, stable or unstable angina, ischemic stroke and transient ischemic attack in patients with dyslipidemia, and thus, this would reduce utilization of costly healthcare services and relevant costs.

\section{Data availability}

The raw data that support the findings of this study are available only for authorized researchers in South Korea and for a limited period due to the information protection law for patient privacy.

Received: 13 September 2021; Accepted: 8 February 2022

Published online: 23 February 2022

\section{References}

1. Reiner, Z. et al. ESC/EAS Guidelines for the management of dyslipidaemias: The Task Force for the management of dyslipidaemias of the European Society of Cardiology (ESC) and the European Atherosclerosis Society (EAS). Eur. Heart J. 32, 1769-1818. https:// doi.org/10.1093/eurheartj/ehr158 (2011).

2. Rader, D. J., Hoeg, J. M. \& Brewer, B. H. Quantitation of plasma apolipoproteins in the primary and secondary prevention of coronary artery disease. Ann. Intern. Med. 120, 1012-1025. https://doi.org/10.7326/0003-4819-120-12-199406150-00008 (1994).

3. Mach, F. et al. 2019 ESC/EAS Guidelines for the management of dyslipidaemias: Lipid modification to reduce cardiovascular risk: The Task Force for the management of dyslipidaemias of the European Society of Cardiology (ESC) and European Atherosclerosis Society (EAS). Eur. Heart J. 41, 111-188. https://doi.org/10.1093/eurheartj/ehz455 (2019).

4. Hana, T. et al. Global atlas of cardiovascular disease 2000-2016: The path to prevention and control. Glob. Heart 13, 143-163. https://doi.org/10.1016/j.gheart.2018.09.511 (2018).

5. Dong, Z. Epidemiological features of cardiovascular disease in Asia. JACC Asia 1, 1-13. https://doi.org/10.1016/j.jacasi.2021.04. 007 (2021).

6. Cardiovascular diseases (CVDs). World Health Organization. https://www.who.int/news-room/fact-sheets/detail/cardiovasc ular-diseases-(cvds). Accessed 13 Feb 2022.

7. Arnett, D. K. et al. 2019 ACC/AHA guideline on the primary prevention of cardiovascular disease: Executive summary: A report of the American College of Cardiology/American Heart Association Task Force on Clinical Practice Guidelines. Circulation 140, e563-e595. https://doi.org/10.1161/cir.0000000000000677 (2019).

8. Pearson, G. J. et al. Canadian cardiovascular society guidelines for the management of dyslipidemia for the prevention of cardiovascular disease in adults. Can. J. Cardiol. https://doi.org/10.1016/j.cjca.2021.03.016 (2021).

9. Rhee, E. J. et al. 2018 Guidelines for the management of dyslipidemia. Korean J. Intern. Med. 34, 723-771. https://doi.org/10.3904/ kjim.2019.188 (2019).

10. Cho, S. M. J. et al. Dyslipidemia fact sheets in Korea 2020: An analysis of nationwide population-based data. J. Lipid Atheroscler. 10, 202-209. https://doi.org/10.12997/jla.2021.10.2.202 (2021).

11. Jerant, A. F., von Friederichs-Fitzwater, M. M. \& Moore, M. Patients' perceived barriers to active self-management of chronic conditions. Patient Educ. Couns. 57, 300-307. https://doi.org/10.1016/j.pec.2004.08.004 (2005).

12. Grundy, S. M. et al. 2018 AHA/ACC/AACVPR/AAPA/ABC/ACPM/ADA/AGS/APhA/ASPC/NLA/PCNA guideline on the management of blood cholesterol: A report of the American College of Cardiology/American Heart Association Task Force on Clinical Practice Guidelines. Circulation 139, e1082-e1143. https://doi.org/10.1161/CIR.0000000000000625 (2019).

13. Dreiher, J. et al. The association between continuity of care in the community and health outcomes: A population-based study. Isr. J. Health Policy Res. 1, 21. https://doi.org/10.1186/2045-4015-1-21 (2012).

14. Hussey, P. S. et al. Continuity and the costs of care for chronic disease. JAMA Intern. Med. 174, 742-748. https://doi.org/10.1001/ jamainternmed.2014.245 (2014). 
15. Pereira Gray, D. J., Sidaway-Lee, K., White, E., Thorne, A. \& Evans, P. H. Continuity of care with doctors-a matter of life and death? A systematic review of continuity of care and mortality. BMJ Open 8, e021161. https://doi.org/10.1136/bmjopen-2017-021161 (2018).

16. Kao, Y. H., Lin, W. T., Chen, W. H., Wu, S. C. \& Tseng, T. S. Continuity of outpatient care and avoidable hospitalization: A systematic review. Am. J. Manag. Care 25, e126-e134 (2019).

17. Haggerty, J. L. et al. Continuity of care: A multidisciplinary review. BMJ 327, 1219-1221. https://doi.org/10.1136/bmj.327.7425. 1219 (2003).

18. Hong, J. S., Kang, H. C. \& Kim, J. Continuity of care for elderly patients with diabetes mellitus, hypertension, asthma, and chronic obstructive pulmonary disease in Korea. J. Korean Med. Sci. 25, 1259-1271. https://doi.org/10.3346/jkms.2010.25.9.1259 (2010).

19. Vogt, V., Koller, D. \& Sundmacher, L. Continuity of care in the ambulatory sector and hospital admissions among patients with heart failure in Germany. Eur. J. Public Health 26, 555-561. https://doi.org/10.1093/eurpub/ckw018 (2016).

20. Dyslipidemia Fact Sheets in Korea. The Korean Society of Lipid and Atherosclerosis. https://www.lipid.or.kr/bbs/index.html?code= fact sheet\&category=\&gubun=\&page=1\&number=896\&mode=view\&keyfield=\&key. Accessed $13 \mathrm{Feb} 2022$. . (2018).

21. Dyslipidemia Fact Sheets in Korea. The Korean Society of Lipid and Atherosclerosis. https://www.lipid.or.kr/file/Dyslipidemia\% 20Fact\%20Sheets\%202020_201125.pdf. Accessed 13 Feb 2022. (2020).

22. Korea National Health \& Nutrition Examination Survey. Korean Center for Disease Control and Prevention. Korean Center for Disease Control and Prevention. https://knhanes.kdca.go.kr/knhanes/sub01/sub01_05.do\#s50201. Accessed 13 Feb 2022.

23. Global Burden of Disease Study 2019 (GBD 2019) Results. Global Burden of Disease Collaborative Network. Institute for Health Metrics and Evaluation. https://ghdx.healthdata.org/gbd-results-tool. Accessed 13 Feb 2022.

24. 2019 National Health Insurance Statistical Annual Report. Health Insurance Review and Assessment Service \& National Health Insurance Service. (2020).

25. National Health Insurance Sharing Service. National Health Insurance Services. https://nhiss.nhis.or.kr/bd/ab/bdaba011eng.do. Accessed 13 Feb 2022.

26. von Elm, E. et al. The strengthening the reporting of observational studies in epidemiology (STROBE) statement: Guidelines for reporting observational studies. PLoS Med. 4, e296. https://doi.org/10.1371/journal.pmed.0040296 (2007).

27. Yang, Y. et al. High-density lipoprotein cholesterol and the risk of myocardial infarction, stroke, and cause-specific mortality: A nationwide cohort study in Korea. J. Lipid Atheroscler. 10, 74-87. https://doi.org/10.12997/jla.2021.10.1.74 (2021).

28. Bice, T. W. \& Boxerman, S. B. A quantitative measure of continuity of care. Med. Care 15, 347-349. https://doi.org/10.1097/00005 650-197704000-00010 (1977).

29. Choo, E. et al. Assessment of the effects of methodological choice in continuity of care research: A real-world example with dyslipidaemia cohort. BMJ Open 11, e053140. https://doi.org/10.1136/bmjopen-2021-053140 (2021).

30. Health Insurance Statistical Yearbook. National Health Insurance Sharing Service. https://nhiss.nhis.or.kr/bd/ad/bdada013cv.do. Accessed $13 \mathrm{Feb} 2022$.

31. Quan, H. et al. Coding algorithms for defining comorbidities in ICD-9-CM and ICD-10 administrative data. Med. Care 43, 1130-1139. https://doi.org/10.1097/01.mlr.0000182534.19832.83 (2005).

32. Roberge, D., Beaulieu, M. D., Haddad, S., Lebeau, R. \& Pineault, R. Loyalty to the regular care provider: Patients' and physicians' views. Fam. Pract. 18, 53-59. https://doi.org/10.1093/fampra/18.1.53 (2001).

33. Khosravizadeh, O., Ahadinezhad, B., Maleki, A., Vosoughi, P. \& Najafpour, Z. Applying the health belief model and behavior of diabetic patients: A systematic review and meta-analysis. Clin. Diabetol. 10, 209-220. https://doi.org/10.5603/DK.2021.0020 (2021).

34. Robertson, R. \& Burge, P. The impact of patient choice of provider on equity: Analysis of a patient survey. J. Health Serv. Res. Policy 16(Suppl 1), 22-28. https://doi.org/10.1258/jhsrp.2010.010084 (2011).

35. Moscelli, G., Siciliani, L., Gutacker, N. \& Gravelle, H. Location, quality and choice of hospital: Evidence from England 2002-2013. Reg. Sci. Urban Econ. 60, 112-124. https://doi.org/10.1016/j.regsciurbeco.2016.07.001 (2016).

36. Nam, J. H. et al. Impact of continuous care on health outcomes and cost for type 2 diabetes mellitus: Analysis using national health insurance cohort database. Diabetes Metab. J. 43, 776-784. https://doi.org/10.4093/dmj.2018.0189 (2019).

37. National Academies of Sciences, Engineering, and Medicine. Health-Care Utilization as a Proxy in Disability Determination. (National Academies Press, 2018).

38. Lin, I. P., Wu, S. C. \& Huang, S. T. Continuity of care and avoidable hospitalizations for chronic obstructive pulmonary disease (COPD). J. Am. Board Fam. Med. 28, 222-230. https://doi.org/10.3122/jabfm.2015.02.140141 (2015).

39. van Walraven, C., Oake, N., Jennings, A. \& Forster, A. J. The association between continuity of care and outcomes: A systematic and critical review. J. Eval. Clin. Pract. 16, 947-956. https://doi.org/10.1111/j.1365-2753.2009.01235.x (2010).

40. Shin, D. W. et al. Impact of continuity of care on mortality and health care costs: A nationwide cohort study in Korea. Ann. Fam. Med. 12, 534-541. https://doi.org/10.1370/afm.1685 (2014).

41. Cheng, S. H., Chen, C. C. \& Hou, Y. F. A longitudinal examination of continuity of care and avoidable hospitalization: Evidence from a universal coverage health care system. Arch. Intern. Med. 170, 1671-1677. https://doi.org/10.1001/archinternmed.2010. 340 (2010).

42. Lin, I. P. \& Wu, S. C. Effects of long-term high continuity of care on avoidable hospitalizations of chronic obstructive pulmonary disease patients. Health Policy 121, 1001-1007. https://doi.org/10.1016/j.healthpol.2017.06.010 (2017).

43. Dossa, A. R., Moisan, J., Guénette, L., Lauzier, S. \& Grégoire, J.-P. Association between interpersonal continuity of care and medication adherence in type 2 diabetes: An observational cohort study. CMAJ Open 5, E359-E364. https://doi.org/10.9778/cmajo.20160 063 (2017).

44. Lee, S. A. et al. Association between continuity of care and the onset of thyroid disorder among diabetes patients in Korea. Int. J. Environ. Res. Public Health 16, 233. https://doi.org/10.3390/ijerph16020233 (2019).

\section{Acknowledgements}

This work was supported by a National Research Foundation of Korea (NRF) grant funded by the Korea government (MSIT) grant number [2020R1A2C1005061]. This study analyzed claims data from the Korean National Health Insurance Service (research management number NHIS-2020-1-380).

\section{Author contributions}

L.I.H., J.E.J., L.J.H. and C.E.Y. wrote the statistical analysis plan, analyzed data, and drafted the paper. C.E.J. analyzed data and prepared Figs. 1, 2, 3, 4, and 5. L.J.H., C.E.Y. and S.L. wrote the main manuscript text and prepared Tables 1, 2, 3, and 4. J.E.J. supervised all statistical analysis process. L.I.H. was a fund holder and supervised all process of this research. All authors read and approved the final manuscript.

\section{Competing interests}

The authors declare no competing interests. 


\section{Additional information}

Correspondence and requests for materials should be addressed to E.J.J. or I.-H.L.

Reprints and permissions information is available at www.nature.com/reprints.

Publisher's note Springer Nature remains neutral with regard to jurisdictional claims in published maps and institutional affiliations.

(c) (i) Open Access This article is licensed under a Creative Commons Attribution 4.0 International License, which permits use, sharing, adaptation, distribution and reproduction in any medium or format, as long as you give appropriate credit to the original author(s) and the source, provide a link to the Creative Commons licence, and indicate if changes were made. The images or other third party material in this article are included in the article's Creative Commons licence, unless indicated otherwise in a credit line to the material. If material is not included in the article's Creative Commons licence and your intended use is not permitted by statutory regulation or exceeds the permitted use, you will need to obtain permission directly from the copyright holder. To view a copy of this licence, visit http://creativecommons.org/licenses/by/4.0/.

(C) The Author(s) 2022 